\title{
Die Quartärforschung im Lichte der modernen Anforderungen der Angewandten Geologie **)
}

\author{
GERD LÜTTIG *)
}

\begin{abstract}
Quaternary, surficial geology, land use, natural resources, energy resources, ore minerals, industrial minerals, water, soil, foundations, environmental geology, regional planning, research, education
\end{abstract}

\begin{abstract}
Kurzfassung: Die Angewandte Geologie, also Wirtschaftsgeologie, Lagerstättenkunde, Ingenieur-, Hydro-, Umwelt-, Planungs- usw. Geologie, bekommt von der Gesellschaft Aufträge und Anforderungen gestellt, die - wenigstens in Mitteleuropa - i. w. mit Lockerablagerungen, vorwiegend quartären Alters, zu tun haben. Die Bedarfsstatistik läßt das klar erkennen.
\end{abstract}

Hingegen zeigt die statistische Betrachtung von Ausbildung und Lehre sowie der Forschungsaktivitäten ein gänzlich anderes Bild. Den Gründen dieser Diskrepanz wird nachgegangen; die Folgerungen werden aufgezeigt.

Abstract: Applied geology, that is industrial, economic and engineering geology, hydrogeology, environmental and planning geology, etc. receive assignments and requirements from society, which - at least in Central Europe - generally have to do with unconsolidated rocks, primarily of the Quaternary. This can be clearly seen in the statistics on demands.

On the other hand, the statistical picture of training and teaching as well as research activitites is quite different. The reasons for this discrepancy are investigated; the consequences are shown.

\section{Die gegenwärtige geopolitische Situation} und die daraus resultierenden Aufgaben für die Angewandte Geologie

Die Geowissenschaften sind seit ihrer Begründung in die Naturwissenschaften eingebettet, und diese bewegen sich gegenwärtig trotz aller Verpflichtung, nach

*) Anschrift des Autors: Prof. Dr. G. LUUTTIG, Ordinarius für Angewandte Geologie an der Universität ErlangenNürnberg, Schloßgarten 5, D - 8520 Erlangen.

**) Nach einem Vortrag auf der 22. wissenschaftlichen Tagung der DEUQUA in Freiburg i. Br. am 10. 09. 1985. der reinen Wahrheit - was immer das auch sein mag - zu suchen, in starkem Maße in eine Rolle, in der sie in enger Beziehung zu den Fragen des Alltags stehen. Da die Probleme, die mit der Erfüllung der LebensGrundbedürfnisse zusammenhängen, ohne Rückgriff auf das Geopotential ( = das durch geologische Gegebenheiten geschaffene Naturraumpotential, vON DANIELS \& LUTTIG 1980) nicht geklärt werden können, sind alle Lösungsversuche ohne Mithilfe der Geowissenschaftler von vornherein aussichtslos. Diese Tatsache ist für uns sowohl Verpflichtung als auch Chance; sie in unseren Zirkeln immer wieder herauszustellen, ist das Hauptanliegen dieser Abhandlung.

Seit dem Zweiten Weltkrieg hat sich die Zahl der Erdenbewohner in einer explosiven Weise, allein zwischen 1950 und 1975 von 2,5 auf 4 Mia. vermehrt. Sie dürfte gegenwärtig 4,6 Mia. betragen. Die Schätzungen für das Jahr 2000 liegen zwischen 5,8 und 6,6 Mia., davon 4,5 bis 5,2 Mia. in den Entwicklungsländern. Dort wird das Bevölkerungsproblem durch die Konzentration in den Großstädten erhöht, Zwangspunkte sind dabei Wasserversorgung und $\mathrm{Ab}$ fallbeseitigung. Der Verbrauch an Naturgütern hat sich, auch durch die Steigerung des Pro-KopfKonsums in den Entwicklungsländern, drastisch erhöht. Wirtschaftlicher Erkenntnis-Umsatz und technische Möglichkeiten haben sich vervielfacht. Die Freisetzung menschlichen Intellekts hat die technischen Grenzen der Zivilisation herausgeschoben. Diese Entwicklung wurde begleitet von einer rasanten $\mathrm{Zu}$ nahme der Anzahl von Informationen, die das Individuum zu verarbeiten hat. Aber es sind nur Teile der Menschheit, die von diesen Innovationen Nutzen hatten, an anderen, die ihr Zivilisationsniveau noch entwickeln müssen, sind sie spurlos vorübergegangen, oder dort sind erhebliche Anstrengungen zur Hebung des Lebensstandards notwendig. 
Kommunikation, verbesserter Güteraustausch, Intensivierung der Handelsbedürfnisse haben zu einer Verkettung geführt, die nicht nur in der Bildung politischer Blöcke, sondern auch in oft quer durch dieselben gehenden wirtschaftlichen Zwängen zum Ausdruck kommt. Dieses bedeutet, daß sowohl Nationen als auch der Einzelne sich in finanzieller, wissensmäßiger, technischer Know-how-Abhängigkeit befinden, wodurch die intellektuelle Selbständigkeit in Frage gestellt wird. Damit verbunden ist die Entstehung einer kulturell-ethnisch-historischen Grauzone, in der die gesunden und natürlichen Demarkationslinien zwischen gewachsenen Gruppen der Menschheit einem gefährlichen Kosmopolitentum weichen.

Das Interesse der Völker liegt in erster Linie in der Verbesserung der Qualität des Lebens. Ein Zustand wird gesucht, in welchem Völker und Individuen ohne Furcht und Angst vor dem Nachbarn leben können und Platz für freie Entwicklung und Kreativität behalten (M'Bow 1982). Disparitäten, Unsicherheit, Verzweiflung und unlösbare Konflikte sind aber noch nicht ausgeräumt. Verzweiflung kann zu Aktionen führen, mittels derer eine geordnete Nutzung der Möglichkeiten unseres Naturraumpotentials unmöglich wird.

Die katastrophale Armut in einigen Regionen hat zur Überbeanspruchung des Naturraumes geführt. Man war und ist dort der Meinung, man müsse das, was man an technischen, ernährungsmäßigen, gesundheitlichen Möglichkeiten nicht besitzt, dadurch wettmachen, daß man die Natur in rücksichtsloser Weise ausbeutet. Naturschätze werden ausgebeutet, um auf einfache Weise ökonomische Vorteile zu erzielen.

Daneben werden weiterhin Instrumente der Zerstörung weiterentwickelt und angehäuft. Durch die Produktion von Waffen werden jedoch fortwährend große Mengen mineralischer Rohstoffe verbraucht. Natürliche Ressourcen, auch geogene, werden vernichtet, die weniger ambitiöse Gruppen für friedliche Zwecke dringend benötigen.

Mit den politisch-strukturellen Änderungen nach 1945 und der Verdichtung des Ost-West- und NordSüd-Handels ist eine Integration einer zunehmenden Anzahl von Nationalökonomien entstanden. Neue Industrien, bedingt durch neue Produkte, haben die Expansion und Entstehung von Produktionszentren in bestimmten Regionen hervorgerufen, die vorher überhaupt nicht für die Industrialisierung vorgesehen waren. Gleichzeitig rief diese technische Entwicklung den Zugriff $\mathrm{zu}$ völlig anderen als den bisher benötigten, aber auch eine Steigerung des Verbrauchs konventioneller Rohstoffe hervor (man denke nur an die Plastik-Industrie!). Einkommens-, Umwelt- und medizinische Veränderungen waren in den entsprechenden Gebieten damit verbunden.

$\mathrm{Zu}$ dieser wirtschaftlichen Umstellung kommt eine gefährliche Acceleration der Ernährungs- und Gesundheits-Situation, die in weiten Teilen der Erde Anlaß zu Besorgnis gibt. Wichtiges Ziel muß die Beseitigung des Hungers in der Welt sein. Die Vermehrung der Agrarproduktion und der Wasserressourcen ist dringlich. Das ist wiederum eine Aufgabe, die ohne geowissenschaftlichen Sachverstand nicht gelöst werden kann.

Über die Ressourcen-Situation der Welt, v. a. im Hinblick auf mineralische Rohstoffe und Energieträger und die Voraussage für deren zukünftige Entwicklung gibt es unterschiedliche Ansichten: Es ist klar, daß einige Nationen wesentlich besser mit Ressourcen versehen sind als andere, so daß es ihnen leichter möglich ist, ihre Lebensbedingungen mit Hilfe dieser Ressourcen zu verbessern.

In einigen Ländern ist hingegen das Geopotential beschränkt. Auch ist die Ausgangslage der Nationen in bezug auf natürliche Risiken und aus der Geo-Situation herrührende Gefahren durchaus unterschiedlich. Dabei gilt zu bedenken, daß einige geologische Gegebenheiten über Leben und Tod der in diesem Raum lebenden Menschen entscheiden können, z. B. der Mangel an landwirtschaftlichem Ertragspotential, Erdbebengefährdung, Überschwemmungen und dgl. Die entsprechenden Populationen sind dadurch in ihren Aktivitäten wesentlich gehandicapt. Aus dieser unterschiedlichen Situation erwächst die Notwendigkeit, erdgeschichtliche Prozesse zu studieren, die Entwicklung in der betreffenden Region zu rekapitulieren und daraus ein zukunftsorientiertes Denken auf der Basis der in der Natur vorhandenen Möglichkeiten zu entwickeln. Zahlreiche Studien dieser Art sind in den letzten Jahren angefertigt worden. Sie sind aber sehr unterschiedlich und heterogen, bedingt durch die angewandten Methoden und auch Ziele und durch den Einfluß z. T. politischer oder zumindest philosophischer Denkweisen, die nicht übereinstimmen. Es ist daher schwer möglich, die Dinge objektiv zu beurteilen.

Fest steht jedoch, von welchem Gesichtswinkel man auch an die Fragen, die die Menschheit jetzt stark beschäftigen, herangeht, folgendes: Alle Bemühungen, ganz gleich welcher Art, werden a $11 \mathrm{e}$ in der modernen Gesellschaft vorhandenen Systeme betreffen müssen. Die Entwicklung e i n e $r$ Population für sich allein ist wegen der starken Zusammenhänge zu anderen Nationen nicht möglich.

Wichtigstes Einzelproblem ist unzweifelhaft die Freisetzung vorhandenen Geop ote ntials. Entwicklung - was immer diese 
Vokabel auch bedeuten mag - bedingt das Vorhandensein eines Potentials, das der Mensch nutzen kann. Dieser Schatz, den die Natur auf Grund der Entstehung der Erdkruste und der räumlichen Verbreitung geologischer Körper an bestimmte Stellen des Planeten (und oft leider $\mathrm{n} \mathrm{u} \mathrm{r}$ an diese) gelegt hat, der den geogenen Teil des Naturraumpotentials, den wir G e o p ot e n t i a 1 nennen, beinhaltet

- Lagerstätten von metallischen und nichtmetallischen Rohstoffen,

- Energieträger, und zwar erneuerbare (wie z. B. geothermische Energie) sowie nicht erneuerbare (wie Erdöl, Erdgas, Kohle, Torf, radioaktive Minerale),

- Grundwasser,

- Böden mit hoher Produktionskapazität,

- geotechnisch vorteilhafte Gegebenheiten (wie z. B. Talsperren-Lokalitäten und Deponiestandorte)

- und andere geogene Situationen.

Das Geopotential ist für die Wirtschaftsentwicklung der Völker - beginnend mit der Verwendung des Flintes als Waffe, über die Entdeckung der Metalle und endend mit dem Einsatz radioaktiver Substanzen als Stromerzeuger - von ausschlaggebender Bedeutung gewesen und wird es immer sein. Die Geologie und die verwandten Wissensgebiete wie Geophysik, Geochemie, Mineralogie, Hydrogeologie und Hydrologie, Bodenkunde sowie Ingenieurwissenschaften, Biologie usf. besitzen nicht nur für die Auffindung und Erforschung des Geopotentials, sondern auch für die Entwicklung von Nutzungsmöglichkeiten eine Schlüsselstellung.

Voran stehen die Fragen:

- Wie ist dieses Geopotential zugänglich zu machen?

- Was bedeutet es wirtschaftlich?

- Beeinflußt es die technologischen Konzepte?

- Wie ist sein Rang in der Gegenwart, wie wird er in Zukunft sein?

- Wo bestehen Chancen, weiteres Naturraumpotential aufzufinden?

\section{Was hat die Quartärforschung mit diesen Fragen zu tun?}

Der Laie und selbst der mit wirtschaftsgeologischen Fragen wenig befaßte Wissenschaftler ist, wenn man auf die Notwendigkeit des Einsatzes geowissenschaftlichen Fachverstandes bei der Lösung von Grundbedürfnisproblemen verweist, gewöhnlich fassungslos.
Fügt man hinzu, daß gerade die Quartärforscher in dieser Hinsicht benötigt werden, erfährt man häufig völliges Unverständnis. Das mag auch daran liegen, $\mathrm{da} ß$ auch heute noch manchem Quartärforscher die Fähigkeit abgeht, sich bei praktischen gutachterlichen Äußerungen verständlich zu äußern, Dinge (wie z. B. petrographische Einzelheiten, stratigraphische $\mathrm{Ab}$ handlungen, Berichte über Flora und Fauna zur Zeit der Entstehung z. B. eines Terrassenkörpers), die den Auftraggeber nicht interessieren, wegzulassen, direkt zur wirtschaftlichen Frage zu kommen. Die Rolle der Erdöl-, Metallerz-, Hydro-, Agro-, Ingenieur-Geologen etc. ist dabei dem Nichtgeologen i. a. eher klar, aber was die Angewandte Quartärgeologie zur Erfüllung der Grundbedürfnisse beizutragen haben soll, wird der Laie nicht gleich begreifen.

Dabei wächst das Verständnis leicht, wenn man auf einige Tatsachen verweist, die die Rolle der quartären, meist aus Lockerablagerungen bestehenden Bildungen für die Menschheit betreffen. Aus diesen Tatsachen seien einige wahllos herausgegriffen:

- $70 \%$ der Menschheit leben in marin beeinflußten Gebieten, meist auf quartären Lockersedimenten.

- Quartäre Sande und Kiese stehen in der Förderstatistik der mineralischen Rohstoffe weltweit an zweiter Stelle hinter den Hart- und Werksteinen (vgl. die Rohstoffschlange, LÜTTIG 1979).

- Für die Tatsache, daß in der Wertstatistik der geförderten mineralischen Rohstoffe die Nichtmetall- die Metallrohstoffe seit 1950 überholt haben und sich ständig weiter über diese erheben (Abb. 1), sind v. a. die quartären Lockersedimente verantwortlich.

- Die gleichen Bildungen sind die wichtigsten für die Versorgung mit Trink- und Brauchwasser herangezogenen Aquifere.

- Das land- und forstwirtschaftliche Ertragspotential in den hauptsächlichen Produktionsgebieten der Erde ist ohne die quartäre Bodenbildung und in mehreren Gebieten ohne quartäre Lockerablagerungen undenkbar (Tschernoseme auf Löß, Marschenböden, Aueböden, Kalkverwitterungsböden etc.):

- Die meisten Hoch-, Tief- und Verkehrswegebaugründungen finden in quartären Lockerablagerungen statt, die Bodenmechanik muß daher häufig auf quartärgeologische Erkenntnisse zurückgreifen.

Die quartären Bildungen sind daher nicht ,der Dreck, der die Geologie verschleiert" (vgl. LÜTTIG 1985). Sie sind vielmehr 


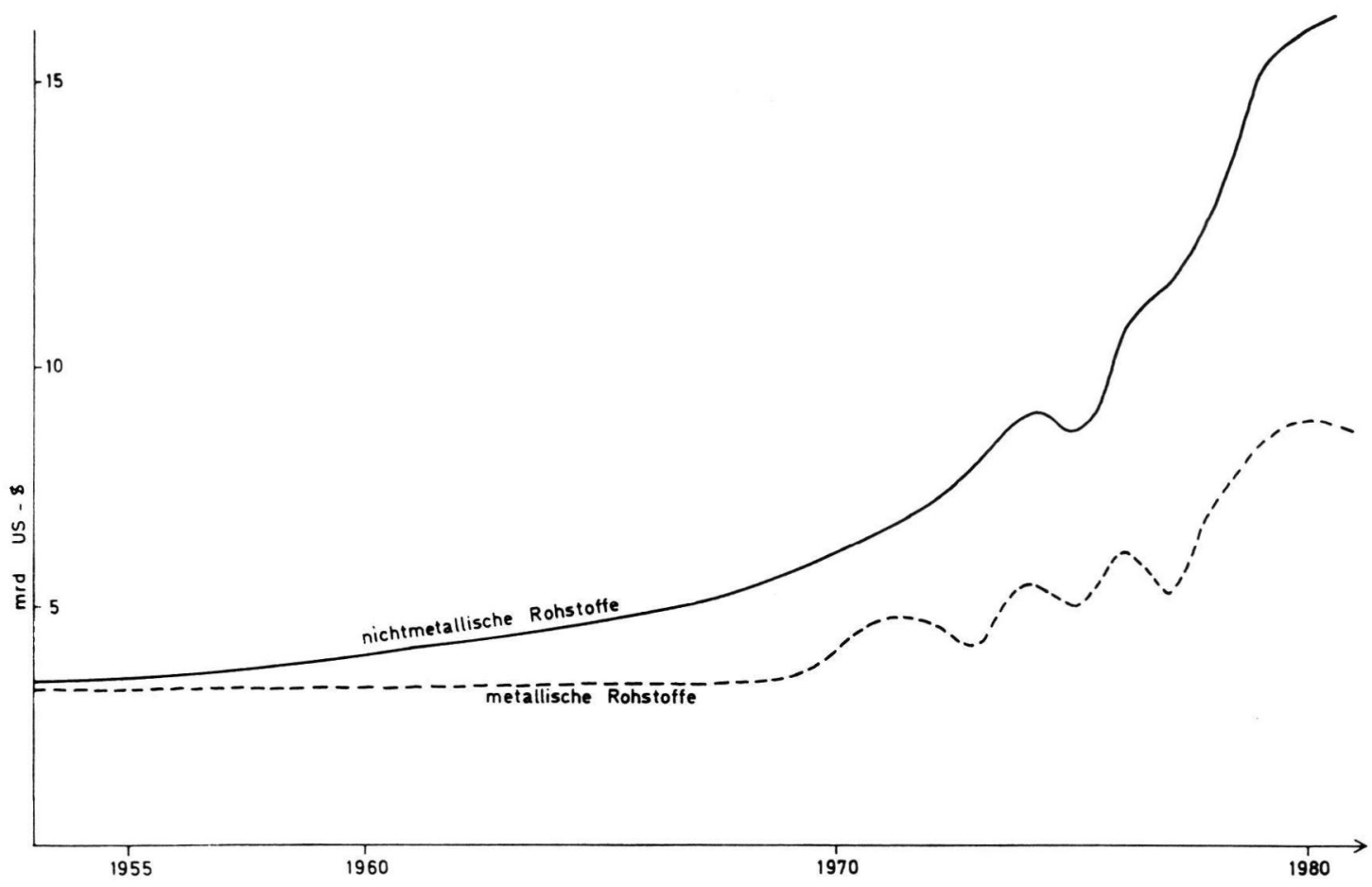

Abb. 1: Produktions-Wertzuwachs der Nichtbrennstoff-Rohstoffe in den USA (nach Unterlagen des USBM).

- von äußerstem wirtschaftlichen Interesse,

- bieten eine Fülle angewandt-wissenschaftlicher Fragestellungen und Aufgaben und

- können daher auch zu einem wachsenden Bedarf an Fachleuten in den einschlägigen Industrien, Wissenschafts- und Verwaltungszweigen führen.

\section{Die Quartärgeologie auf dem Sektor der mineralischen Rohstoffe und Energieträger}

Vor allem in den Entwicklungsländern hängen viele der praktischen Fragen mit der Quartärgeologie zusammen. Und in den Industrienationen ist dieses Feld noch längst nicht abgegrast. Dazu einige Beispiele aus den verschiedenen Bereichen der Angewandten Geologie:

\section{Energieträger}

Quartäre Kohlengesteine sind in viel größerem Maße als bisher zur Deckung der Energielücken heranziehbar; dazu fehlt in großen Teilen der Erde noch die hinreichende Explorationsdichte. Zahlreiche Neufunde an quartären $-z$. T. auch pliozänen; man sollte' hier den stratigraphischen Unterschied nicht zu wichtig nehmen - Braunkohlen und Torfen bezeugen das
(Megalopolis/Griechenland, LÜTTIG \& MARINOS 1962; Philippi/Griechenland, MELIDONIS 1981, CHRISTANIS 1982; Elbistan/Türkei, GolD \& LUUTTIG 1972; Pietrafitta/Italien, Padul/Spanien, FLORSCHÜTZ \& MENENDEZ AMOR 1962; Oued Nja/Marokko, LÜTTIG, unveröff.; Hula-See/Israel, PICARD 1963). Neben den großen unexplorierten Braunkohlen-Hoffnungsgebieten in den alpidischen Orogenen stehen Torflagerstätten des Holozäns, die in einigen Ländern der Erde, obwohl ihr Anteil an den Gesamtreserven der Kohlengesteine nur knapp $1 \%$ beträgt (vgl. Abb. 2), von erheblicher Bedeutung für die Energieversorgung sind (Irland, Finnland, Kanada, Sowjetunion) und in anderen, auch tropischen Ländern noch verstärkt in Einsatz kommen könnten ( $z$. B. anstelle von Holzkohle in Brasilien, Jamaica, Argentinien, in Burundi, Indonesien). Auch die rezente Statistik zeigt im Vergleich von Reserven und Ressourcen (Abb. 3) die großen Chancen für die Erweiterung der Reserven bei den Braunkohlen.

Interessante, bisher ungeahnte Möglichkeiten bieten rezente Methanansammlungen im Kiwu-See (TIETZE et. al. 1980), sicherlich sind sie nicht der einzigste Fall dieser Art.

Große Chancen sind in der Nutzung der erneuerbaren geothermischen Energie, die ja z. T. an quartäre Vulkanitregionen gebunden sind, zu erblicken. Daß hier 


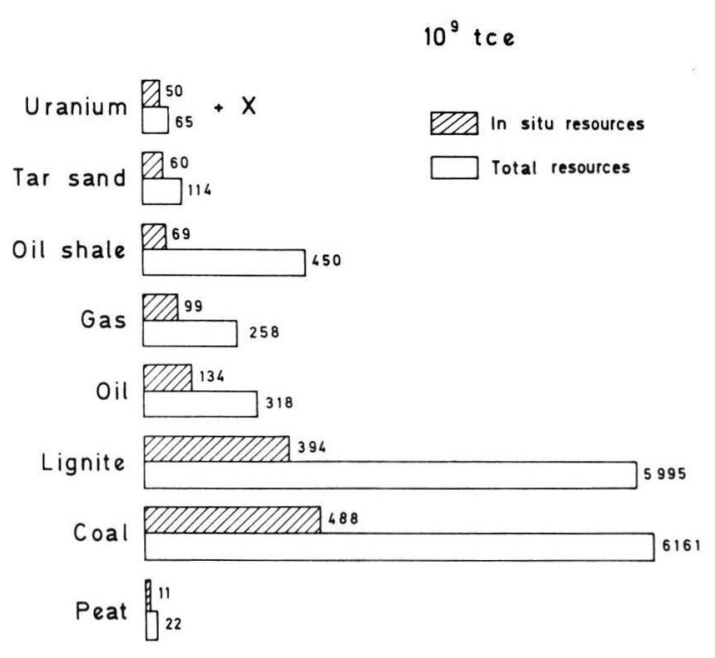

Abb. 2: In-situ- und Total-Ressourcen der nicht erneuerbaren Energieträger, umgerechnet in Steinkohleneinheiten (tce). Beim Uran handelt es sich um eine sehr zurückhaltende Schätzung auf der Basis einer hohen Preisklasse (nach LÜTTIG, 1983).

v. a. in den Entwicklungsländern Afrikas und Lateinamerikas nur erst ein Anfang gemacht wurde, ist zu beklagen. Gerade für die Deckung von Energie-Angebotslücken, auch im Hinblick auf den Energiebedarf bei der Verarbeitung von Armerzen, der Düngemittelherstellung und für Irrigationsvorhaben ist dieses Angebot nicht zu unterschätzen. Es ist völlig verfehlt anzunehmen, daß die geothermische Prospektion, die i. w. in den Händen der Geophysiker und Petrologen liegt, ohne die Beratung der Quartärforscher auskommt.

Hinzu kommen die Möglichkeiten des Einsatzes von Biomasse zur Biokonversion, v. a. in den tropischen Forstgebieten; bodenkundlicher und hydrogeologischer Sachverstand wird auch hier gesucht.

Nicht zu übersehen ist der Energieträger Wasser. Auch hier gibt es ein für die Quartärforscher interessantes Projekt. Zwei Schweizer Wissenschaftler, KOLLBRUNNER \& STAUBER (1973), haben vorgeschlagen, im Polarkreis eine Art von Pumpspeicherwerk einzurichten. Subglaziäres Schmelzwasser aus hochliegenden Gletscher- und Inlandeiskörpern soll danach zur Energiegewinnung benutzt werden. Freilich ist diese Idee noch weit von wirtschaftlichen Bedingungen entfernt.

Reelleren Hintergrund hat das Wasserkraft (Staudamm)-Potential, und es ist schon merkwürdig, daß dieses die geringsten Stromerzeugungskosten bedingende Geopotential in seinem weltweiten Angebot noch nicht einmal verläßlich bekannt ist. So spricht

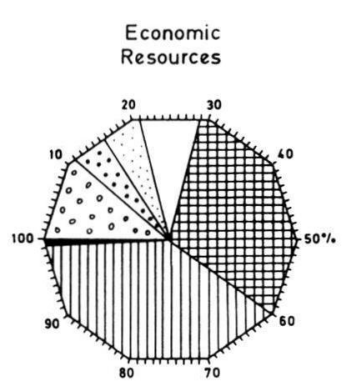

$\because$ Oil

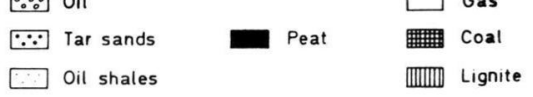

Abb. 3: Prozentverteilung der Ökonomischen und Total-Ressourcen der nicht erneuerbaren Energieträger der Erde (nach ArCHER, LútTig \& SNEZHKo, 1987).

die EG von 2,34 TW (1 TW $=1.000 .000 \mathrm{MW})$ an Wasserkraftpotential der Erde, von denen nur 1,3 TW ausgenutzt werden; die Weltenergiekonferenz berechnete ein Potential von 2,26, BISWAS (1981) ein solches von 3,2 TW. Nach Ansicht des Autors ist es eine dringende Aufgabe von Geomorphologen, Hydrologen, Talsperrengeologen und Wasserwirtschaftlern, in Form von Länderstudien die auf der Erde vorhandenen Möglichkeiten abzuschätzen.

\section{Metallische Rohstoffe}

In Metallerz-Lagerstättenkundler-Kreisen hört man sehr häufig die Behauptung, für die Bildung des Metall-Geopotentials sei einzig und allein das präkambrische Kristallin, wirtschaftlich gesehen, relevant. So wird dort z. B. gesagt, der gesamte Mineralreichtum Afrikas sei an die präkambrischen Schilde gebunden. Das ist schlichtweg falsch. Zum einen zeigt die Rohstoffstatistik, daß Metallrohstoffe nur deswegen als ökonomisch so bedeutend erscheinen, weil die Nichtmetallrohstoffe nur selten erfaßt sind. Dabei liegt das Eisen als der mengenmäßig wichtigste Metallrohstoff statistisch erst an sechster, rechnet man das Wasser dazu, an siebter Stelle. In der Wertstatistik ist Kupfer an der Spitze der Metallrohstoffe erst Rohstoff Nr. 9 bzw. 10, da der Bauxit als Ursprung der Tonerde - er liegt an siebter bzw. achter Stelle - zur Hälfte zu den nichtmetallischen Rohstoffen gezählt werden muß.

Zum zweiten steigt in Anbetracht der Zunahme der Förderkosten bei den Bergbaufirmen die Neigung, im Tagebau gewinnbare und Massenerze den nur untertage gewinnbaren und Gangerzen, von Ausnahmen abgesehen, vorzuziehen. Dadurch rücken die ohnehin 
auf dem Sektor der Zirkon-, Rutil-, Ilmenit-, Zinn-, Monazitgewinnung führenden quartären Seifenlagerstätten in das Blickfeld, und hier ist auch eine Erweiterung auf die Chromitseifen nötig. Überraschend ist festzustellen, daß auf diesem Gebiet wie bei den Gold- und Diamantseifenlagerstätten quartärgeologisch-sedimentologischer Sachverstand in beschämender Weise unterentwickelt ist, falls die Bergleute, wie auf diesem Gebiet besonders üblich, Geologen überhaupt zu Rate ziehen (woran überwiegend die Geologen selbst schuld sind, weil sie keinen brauchbaren Rat zu bieten haben).

\section{Nichtmetall-Rohstoffe}

Dieser Sektor ist von den Geowissenschaftlern erst in den letzten Jahren als wichtig entdeckt worden.

Unter den Nichtmetall-Rohstoffen, besser IndustrieMinerale und -Gesteine zu nennen, ist der Anteil quartärer Bildungen beachtlich. Das zu veranschaulichen, wird in Tabelle 1 versucht. Neben Ubiquisten in der stratigraphischen Tabelle fallen ausgesprochen in bestimmten geologischen Systemen verbreitete Rohstoffe auf.

Daß ihre Prospektion, Kartierung, Lagerstättenbewertung und technische Untersuchung im Grunde ohne geowissenschaftlichen Sachverstand nicht möglich ist, sehen zwar sogar manche Nichtquaternaristen, Bergleute und Unternehmer ein, vor allem wenn ihnen eklatante Fehlentscheidungen, finanzielle Verluste oder große technische Schwierigkeiten zu dieser Einsicht verholfen haben. Daß

- zur Beurteilung eines Kieslagerstättengebiets Kenntnisse über Sortierungs-, Abrieb-, Klassierungs-Vorgänge,

- zur Auffindung der für den betreffenden Zweck technisch am besten geeigneten Bentonit-Varietät eine genauere Vorstellung über die geochemische Veränderungen bewirkenden Vorgänge, wie z. B. auch die Geschichte mariner Strandterrassen sowie die Pedogenese und

- zur Ausarbeitung des günstigsten Abbauplanes einer Quarzsandlagerstätte auch das Wissen über den Beanspruchungsplan der quartären Tektonik gehören können, wird dem betreffenden Unternehmer ebenfalls zumeist erst nach einem wirtschaftlichen Rückschlag einsichtig. Ein auf dem Gebiet der känozoischen Industrie-Gesteine tätiger Lagerstättenkundler ohne eine verläßliche Grundausbildung sowohl auf dem Gebiete der allgemeinen als auch der stratigraphischen Quartärgeologie ist verloren.

Ein anderer Aspekt bedarf der Erwähnung: Die Angewandte Quartärgeologie ist besonders wichtig (nicht nur) für die Lagerstättenfragen in Entwicklungsländer. Aus dieser Erkenntnis heraus war die Gründung des von ROLAND PAEPE geleiteten International Training Centre for Applied Quaternary Geology (IFAQ) an der Freien Universität in Brüssel eine sehr kluge Entscheidung.

In den Entwicklungsländern wird nämlich von den Entscheidungsträgern bei wirtschaftlichen, mit Rohstoffen zusammenhängenden Projekten häufig ein Fehler gemacht: Die Tendenz zu Großprojekten mit langen, durch Prospektion und Exploration, Aufbereitungsversuche, Feasibility-Studien bedingten Vorlaufzeiten, hohem Kapitalaufwand für Investitionen und sehr spätem Cash-Flow herrscht vor. Hier spielen auch Ehrgeiz und Profitsucht eine Rolle. Oft stehen die Projekte nicht auf der Basis sicherer geologischlagerstättenkundlichen Nachweises.

So sind in mehreren Ländern Projekte angefangen und nie realisiert, Verarbeitungsbetriebe ohne Vorhandenseins der Rohstoffe bzw. zusätzlich nötiger Infrastruktur errichtet, z. T. Hütten und Aufbereitungsanlagen direkt auf die Lagerstätte gesetzt worden.

Dagegen wäre sinnvoll gewesen, mit kleinen Projekten zu beginnen, die man mit geringem Finanzaufwand, brauchbarem Know-how aber raschem Cashflow hätte durchführen können. Dazu gehören v. a. Projekte für die Baustoffwirtschaft, keramische Industrie, Glasindustrie, Gips- und Zementwerke, und in vielen Fällen ist gerade hier die Möglichkeit, quartäre Bildungen abzubauen, gegeben. Die Erfahrung zeigt, $\mathrm{da} \beta$ derartige Vorhaben, selbst in den LLCDs (Least Developed Countries) bzw. MSACs (Most Seriously Affected Countries) sich zu den günstigsten auf dem Rohstoffsektor entwickelt haben. In diesem $\mathrm{Zu}$ sammenhang ist v. a. die Arbeitsweise der UNIDO (UNITEd NATIONS INDUSTRIAL DEVELOPMENT ORGANISATION, Wien) mustergültig.

\section{Hydrogeologische Fragen}

In einer Zeit, in der durch

- die natürliche Gewässergüte-Beeinträchtigung (durch Brackwasserbeeinflussung von FlußUnterläufen, Ablaugung von Salz- und Gipsgesteinen in Flußsystemen, krenogenem MineralEintrag, Eutrophierung von Seen, durch Moorwässer etc.)

und

- die anthropogene Kontamination (Einleitung von Abwässern aller Art, Eintrag radioaktiver und anderer Emissionen, Kühlwässer, Beeinflussung durch Deponien jeglicher Form und Zusammensetzung, Verfrachtung von Düngemitteln usw.) 
Tab. 1: Schwerpunkte der stratigraphischen Provenienz der wichtigsten Industrie-Minerale und -Gesteine

Industrie-Mineral

oder -Gestein
Stratigraphisches System

\begin{tabular}{|c|c|c|c|c|c|}
\hline Quartär & Tertiär & Kreide & Trias-Jura & $\begin{array}{l}\text { Permo- } \\
\text { karbon }\end{array}$ & $\begin{array}{c}\text { Altpaläo- } \\
\text { zoikum }\end{array}$ \\
\hline
\end{tabular}

\begin{tabular}{|c|c|c|c|c|c|c|c|c|}
\hline \multirow{15}{*}{ 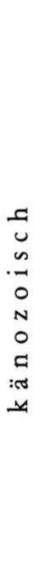 } & Kies und Sand & $\left.\mathrm{X}^{*}\right)$ & $\left.\mathrm{O}^{* *}\right)$ & . & . & & & \\
\hline & Quarzsand & $\mathrm{O}$ & $\mathrm{x}$ & $\mathrm{O}$ & . & & & \\
\hline & Quarzkies & 0 & $\mathrm{X}$ & . & & & & \\
\hline & Ilmenit, Rutil, Zirkon & $\mathrm{X}$ & $\mathrm{O}$ & & & . & . & · \\
\hline & Ton, grobkeramisch & $\mathrm{X}$ & $\mathrm{X}$ & $\mathrm{O}$ & $\mathrm{O}$ & . & & \\
\hline & Ton, feinkeramisch & $\mathrm{O}$ & $\mathrm{X}$ & . & . & & & \\
\hline & Ton, feuerfest & . & $\mathrm{X}$ & $\mathrm{O}$ & . & & & \\
\hline & Kaolin & . & $\mathrm{X}$ & $\mathrm{O}$ & . & & & \\
\hline & Bentonit & $\cdot$ & $\mathrm{X}$ & $\mathrm{O}$ & . & & & \\
\hline & Blähton-Rohstoffe & $\mathrm{X}$ & $\mathrm{X}$ & $\mathrm{X}$ & $\mathrm{x}$ & . & . & \\
\hline & Seltene-Erden-Rohstoffe & $\mathrm{X}$ & $\mathrm{O}$ & . & & & & \\
\hline & Bims, Perlit & $\mathrm{X}$ & $\mathrm{O}$ & . & & & & \\
\hline & Kieselgur, Kieselerde & $\mathrm{X}$ & $\mathrm{X}$ & $\mathrm{x}$ & . & & & \\
\hline & Bor-, Brom-, Jod-Rohstoffe & $\mathrm{X}$ & $\mathrm{O}$ & . & . & $\mathrm{X}$ & & \\
\hline & Torf & $\mathrm{X}$ & & & & & & \\
\hline \multirow{8}{*}{ 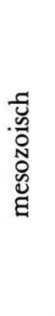 } & Bauxit, Tonerde & . & $\mathrm{X}$ & $\mathrm{X}$ & $\mathrm{O}$ & & & \\
\hline & Phosphat & . & $\mathrm{X}$ & $\mathrm{X}$ & . & & & \\
\hline & Strontium-Rohstoffe & & . & $\mathrm{X}$ & $\mathrm{x}$ & . & & \\
\hline & Flint & & . & $\mathrm{X}$ & . & & & \\
\hline & Zementkalk & . & $\mathrm{O}$ & $\mathrm{X}$ & $\mathrm{x}$ & $\mathrm{O}$ & . & \\
\hline & Industriekalk & . & $\mathrm{O}$ & $\mathrm{x}$ & $\mathrm{X}$ & $\mathrm{O}$ & $\mathrm{O}$ & \\
\hline & Dolomit & & $\cdot$ & $\mathrm{O}$ & $\mathrm{X}$ & $\mathrm{X}$ & $\mathrm{X}$ & . \\
\hline & Magnesit & & & $\cdot$ & $\mathrm{X}$ & $\mathrm{O}$ & $\mathrm{O}$ & \\
\hline \multirow{8}{*}{ 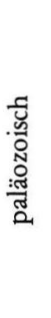 } & Hart- und Werksteine & . & $\mathrm{X}$ & $\mathrm{X}$ & $\mathrm{O}$ & $\mathrm{O}$ & $\mathrm{O}$ & $\mathrm{O}$ \\
\hline & Quarzit, feuerfest & & . & . & $\mathrm{X}$ & $\mathrm{O}$ & $\mathrm{X}$ & $\mathrm{O}$ \\
\hline & Schwerspat & . & $\mathrm{O}$ & . & $\mathrm{X}$ & $\mathrm{x}$ & $\mathrm{X}$ & $\mathrm{O}$ \\
\hline & Flußspat & $\mathrm{O}$ & $\mathrm{O}$ & . & $\mathrm{X}$ & $\mathrm{X}$ & $\mathrm{X}$ & $\mathrm{X}$ \\
\hline & Gips und Anhydrit & $\mathrm{O}$ & $\mathrm{x}$ & . & $\mathrm{X}$ & $\mathrm{X}$ & . & \\
\hline & Stein- und Kalisalz & . & $\mathrm{O}$ & . & $\mathrm{O}$ & $\mathrm{X}$ & . & \\
\hline & Schwefel & $\mathrm{O}$ & $\mathrm{X}$ & . & $\mathrm{O}$ & $\mathrm{X}$ & $\cdot$ & \\
\hline & Talk etc. & & $\mathrm{O}$ & . & $\mathrm{O}$ & $\mathrm{x}$ & $\mathrm{x}$ & $\mathrm{X}$ \\
\hline \multirow{5}{*}{ 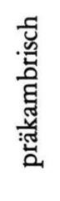 } & Chromit & $\mathrm{O}$ & . & & & $\mathrm{O}$ & $\mathrm{O}$ & $\mathrm{x}$ \\
\hline & Graphit & & & & & $\mathrm{O}$ & $\mathrm{O}$ & $\mathrm{X}$ \\
\hline & Abrasiva & . & . & . & . & . & $\mathrm{O}$ & $\mathrm{X}$ \\
\hline & Glimmer & & & & . & $\mathrm{O}$ & $\mathrm{O}$ & $\mathrm{x}$ \\
\hline & Apatit & & . & . &. & $\cdot$ & $\cdot$ & $\mathrm{X}$ \\
\hline \multirow{4}{*}{$\begin{array}{l}\frac{5}{5} \\
. \frac{0.0}{3} \\
\frac{0}{3} \\
\frac{0}{3}\end{array}$} & Nephelin, Phonolith & $\mathrm{O}$ & $\mathrm{O}$ & . & . & . & $\mathrm{O}$ & $\mathrm{O}$ \\
\hline & Gangquarz & $\cdot$ & $\mathrm{O}$ & . & $\mathrm{O}$ & $\mathrm{O}$ & $\mathrm{O}$ & $\mathrm{O}$ \\
\hline & Feldspat & $\mathrm{O}$ & $\mathrm{O}$ & . & $\mathrm{O}$ & $\mathrm{O}$ & $\mathrm{O}$ & $\mathrm{X}$ \\
\hline & Diamanten & $\mathrm{X}$ & $\mathrm{O}$ & . & . & . & $\mathrm{X}$ & $\mathrm{X}$ \\
\hline
\end{tabular}

*) besonders häufig **) häufig 
Oberflächenwasser für die Versorgung der Menschheit mit Trink- (und auch Brauch-) Wasser in zunehmendem Maße an Eignung verlieren, wird gesteigert auf das Grundwasser rückgegriffen. Seit der von JOHN SNOW anläßlich der Choleraepidemie in den USA (1849) gewonnenen Erkenntnis, daß die Verhinderung von Seuchen in dem Maße besser gelingt, in welchem Grundwasser vermehrt zum Einsatz kommt, ist der Anteil des Grundwassers an der Wasserversorgung in den entwickelten Ländern auf 70-90\% gestiegen.

Diese Tendenz greift glücklicherweise auch auf die Entwicklungsländer über, obwohl dort der Anteil des Grundwassers im einzelnen sehr unterschiedlich ist.

Der größte Teil dieses Grundwassers stammt aus Lockergesteinsaquiferen, schätzungsweise $80 \%$. Diese Aquifere dürften i. w. Sande und Kiese des Quartärs sein.

Für die Erkundung und Nutzung dieser Lockergesteinsaquifere kommt der Geologe ohne gründliche Kenntnis der Quartärgeologie nicht aus. Die vom nutzbaren Porenraum, mithin indirekt von der Korngrößenverteilung, Kompaktion, der Kornbindung, Kornform, von der Mächtigkeit der Ausbildung und Durchlässigkeit der Deckschichten, damit des Rücklage-Anteils der Versickerung und überhaupt dem Bau der Grundwasserlandschaft abhängige Ergiebigkeit des Aquifers ist exakt nur zu ermitteln auf der Basis einer gründlichen Kenntnis über Flußgeschichte, Stratigraphie, Sedimentologie, u. U. auch der Geröllanalyse und -morphometrie, vielleicht auch der jungen Tektonik im betreffenden Grundwasser-Einzugsgebiet. Wer hier nach Grundwasser sucht, ist blind, wenn er nicht weiß, wie sich die Grundwasserlandschaft entwickelt hat.

Wer z. B. in einer Sanderlandschaft Grundwasser exploriert, wird wissen wollen, in welcher Richtung der Sander geschüttet wurde, wo die grobklastische Sanderwurzel liegt und wie diese an Infiltrationsgebiete angeschlossen ist.

Wer in einem ariden Flußgebiet nach Grundwasser sucht, muß angesichts der Tatsache, daß hier die Flüsse das Grundwasser nähren, nach verborgenen, unter Umständen vom rezenten Flußsystem entfernten Flußläufen suchen, in die die fließende Welle Oberflächenwasser einspeist und aus deren Kiesen sie einen hervorragenden Aquifer machen kann (Beispiel: New Valley am Nil).

Und im Falle einer Stockwerkbildung in einer pleistozänen Grobklastika-Serie, die durch eine oder mehrere aus Tonen, Seemergeln, Torfen bestehende Aquicluden getrennt ist, kommt es auch darauf an, gründlich über Stratigraphie, Fazieskunde, Paläogeographie der entsprechenden Thermomere bescheid zu wissen.
In solchen Fällen bewährt sich in besonderem Maße die bei den Quartärforschern eingefahrene Zusammenarbeit zwischen Geologen, Geomorphologen, Sedimentologen, Paläobotanikern, Paläozoologen und natürlich den Geophysikern, Hydrochemikern, Hydraulikern, Klimatologen, Ingenieuren etc.

Von einer systematischen Hinwendung der Quartärforschung zu der praktischen Hydrogeologie können wir aber leider noch keinesfalls sprechen. Hinzuzufügen ist, daß eine Reihe von Angeboten der Wasserwirtschaft von den Quartärforschern nicht richtig angenommen worden ist und daß wegen mangelnder praktischer Ausbildung - siehe die Eingangsbemerkung - noch zu viele, v. a. junge Kollegen nicht in der Lage sind, sich technisch verständlich zu artikulieren.

Was die Entwicklungsländer anbelangt, in denen der Einsatz quartärgeologisch geschulter Hydrogeologen verstärkt werden muß, kann hinzugefügt werden, daß es dort auch darum geht, alte Techniken der Grundwasserentnahme, wie sie v. a. aus dem Nahen Osten, Afrika, dem zentralasiatischen und ostasiatischen Raum bekannt sind, wieder zur Geltung zu bringen.

Grundwasser wird dort auch für die Industriealisierung und Entwicklung der Rohstoffwirtschaft benötigt; seine Rolle für die Steigerung der bewässerbaren Flächen, v. a. in den Least Developed Countries ist nicht zu unterschätzen, v. a. wenn deren Geopotential i. W. auf die Bodenfruchtbarkeit beschränkt ist.

Die Steigerung der Grundwasserproduktion in den Entwicklungsländern wird gegenwärtig noch durch einige Faktoren behindert, von denen die wichtigsten wie folgt genannt werden können:

1) In vielen Ländern fehlt noch eine exakte Abschätzung des Wasserbedarfs.

2) Mehrerenorts ist keine Übersicht über das Angebot an (Oberflächen- und) Grundwasser vorhanden. Die nötigen Institutionen, die hydrogeologische Karten und Bilanzen entwickeln können, fehlen z. T. noch.

3) Es fehlen z. T. auch noch wasserwirtschaftliche Pläne zur Förderung und Verteilung ermittelten Potentials, v. a. im Hinblick auf die Bewässerung.

4) Das technische Inventar an Bohrgeräten, Ausbaumaterial, Pumpen, Verteilungssystemen ist in vielen Ländern äußerst mangelhaft; z. T. fehlen moderne Ausrüstung und Management völlig.

Hier liegt eine der größten Aufgaben und Chancen der Industrienationen für die Entwicklung der armen Nationen. Diese Aufgabe ist von den internationalen Organisationen voll erkannt worden (vgl. UN-Bericht 
1976), und große Mittel werden für die Erfüllung eingesetzt. Auch hier fehlt aber eine systematische Beteiligung von Quartär-Hydrogeologen.

\section{Das Boden-Potential}

Wie in Kapitel 1 deutlich zu machen versucht wurde, ist die Produktion, Bereitstellung und Verteilung ausreichender Mengen von Nahrungsmitteln eine der Hauptaufgaben der modernen Zivilisation. Trotz aller futuristischer Äußerungen einiger Fachleute und Pseudoexperten, nach denen bei der Produktion von Vegetation mit Wasser, Düngemitteln und einer Verankerung für die Pflanzen, z. B. durch den Boden, auszukommen wäre - in Art von Hydrokulturen -, kommt man bei Anwendung ausgewogenen Sachverstandes nicht darum herum, daß ebenso, wie die $\mathrm{H}$ a u t das größte und wichtigste Organ für den Menschen ist, der $\mathrm{B}$ ode $\mathrm{n}$, die $\mathrm{Erdh}$ a u t, die unersetzbare Berührungsfläche zwischen Litho-, Bio-, Hydro- und Atmosphäre bleibt, welche allein gesunde und natürliche Bedingungen für die Umsetzung von Geopotential für das Bios garantiert.

Bodenbildung ist ein auf der rezenten Verwitterung und Umsetzung des Lithos beruhender Vorgang. Die Verwurzelung der Bodenkunde in der Quartärgeologie ist daher unverkennbar. Die Grenze zwischen Pedologie, Mineralogie, Chemie, Physik, Sedimentologie, Quartärgeologie, Hydrologie, Biologie und Klimatologie auf der einen und Land-, Forst- und Ernährungswissenschaft auf der anderen Seite ist hier fließend. Auch die Bodenkunde ist aufgefordert, als angewandte Geowissenschaft an der Lösung der dringenden zivilisatorischen Fragen mitzuwirken, und zwar nicht nur auf Anfrage sondern aktiv, vorausschauend, prospektiv. Das gilt zwar auch in den Zivilisationsgebieten mit hohem Ertrag und Überproduktion, mehr aber in den Ländern, in denen Millionen an Hunger leiden.

Einige der Experten, die von der Möglichkeit der Entwicklung solcher Länder träumen, haben meist den Mineral-Rohstoffteil in bezug auf die Gesamtchance im Auge. Mehrere Entwicklungsländer sind aber mit mineralischen Bodenschätzen so schlecht ausgestattet, daß man nur wünschen kann, diese Länder möchten doch dann ein mindestens brauchbar hohes landwirtschaftliches (und auch forstwirtschaftliches) Potential besitzen, um auf diesem Wege die Grundlagen für einen erträglichen Lebensstandard zu schaffen. Bei dieser Überlegung wird selbstverständlich richtig impliziert, daß eventuell vorhandene ungünstige Produktionsstrukturen oder ,rückständige” — man sollte als Europäer bei einem solchen Wort vorsichtig sein! - Techniken noch nichts über das wahre Potential aussagen. In der Tat sollte in diesem Zusammenhang das Gebiet der Anbaumethoden und Boden-Bearbeitungs-Verfahren zurückstehen und der Sektor der natürlichen Ressourcen im Vordergrund stehen.

Die Chancen der Entwicklungsländer im Hinblick auf die Kapazität ihrer Böden sind in erster Linie vom Ausgangsgestein, Klima und, damit zusammenhängend, Wasserhaushalt abhängig. Und hier liegen die Barrieren für viele Entwicklungsversuche. Was die forstwirtschaftliche Produktivität anbelangt, so sollte sich der Industrielandexperte auch hier hüten, Standpunkte der Forstkultur aus den gemäßigten Zonen leichtfertig auf die Entwicklungsländer zu übertragen. WECK (1962) wies daher mit Recht darauf hin, daß die Grundregeln der Forst- und Holzwirtschaft im Rahmen der Gesamtwirtschaft in den Entwicklungsländern der Tropen und Subtropen genau so wie in den Ländern alter Forstkultur in der gemäßigten Zone gelten. Auch die Forderung nach einem ,,multiple use of forestlands", die der Weltforstkongreß in Seattle 1960 aufstellte, gilt für die Entwicklungsländer. Darüber hinaus kommt es auf spezifische Erfahrungen an, wie die folgenden:

- Der Anteil der Erträge aus Forstwirtschaft am Sozialprodukt, der in den alten Industrieländern Zentraleuropas um den bescheidenen Wert von 0,2 bis $1,5 \%$ pendelt, kann in den für Forstwirtschaft überhaupt tauglichen Entwicklungsländern wesentlich höhere Werte erreichen. In den waldreichen Entwicklungsländern vorliegende Möglichkeiten forstlicher Erzeugung sind jedoch erst zu einem wesentlich geringeren Anteil ausgenutzt als in Mittel- und Westeuropa.

- In den tropischen Entwicklungsländern spielt die Erhaltung oder Wiederherstellung von Baumbewuchs zur Sicherung protektiver Funktionen in der fortlaufend dichter besiedelten Kulturlandschaft in der Regel eine noch wesentlich größere Rolle als bei uns. Vor allem in den feuchten Tropen können sehr große Flächen mit tiefgehend mineralisch verarmten, tonarmen Böden überhaupt nur unter Mitwirkung von Baumbewuchs dauernd produktiv erhalten werden.

- Trotz der für Wirtschaft und Raumordnung besonders großen Bedeutung pfleglicher Forstwirtschaft in den Tropen ist es hier besonders schwierig, eine solche auf großer Fläche in Gang zu bringen. Ursache hierfür sind die in der Regel erst später eingehende und relativ bescheidene Rendite aus Investierungen in der Forstwirtschaft und die Notwendigkeit, den Aufbau einer Forstwirtschaft im Zuge einer überlegten Raumordnung und mit Übergang zu modernen Formen intensiver Landwirtschaft zu vollziehen. Hierfür liegen meist nur unzureichende Ansätze vor. 
Tab. 2: Landwirtschaftliche Produktion ausgewählter Nutzpflanzen in den Entwicklungsländern und Industrieländern (nach VON BLANCKENBURG \& CREMER, 1971, aus LÜTTIG, 1978)

$\begin{array}{cccc}\begin{array}{c}\text { Welt } \\ \text { insgesamt }\end{array} & \begin{array}{c}\text { Welt } \\ \text { durchschnitt }\end{array} & & \\ 1965 & & \text { niedrig } & \text { hoch } \\ \text { Mill. } \mathrm{t} & & & \end{array}$

\begin{tabular}{|c|c|c|c|c|c|}
\hline Weizen & 266 & 12 & 3 Libyen & & Dänemark \\
\hline Reis & 254 & 20 & 13 Philippinen & 62 & Spanien \\
\hline Mais & 226 & 23 & 3 Libyen & 43 & USA \\
\hline Gerste & 105 & 15 & 4 Libyen & 40 & Holland \\
\hline Hirsen & 78 & 10 & 2 Somalia & 39 & Italien \\
\hline Hafer & 47 & 15 & 7 Algerien & 38 & Dänemark \\
\hline Roggen & 35 & 13 & 11 Chile & 30 & Dänemark \\
\hline Kartoffeln & 284 & 121 & 50 Äthiopien & 290 & Holland \\
\hline Süßkartoffeln und Yam & 134 & 83 & 16 Sudan & 200 & Ägypten \\
\hline Maniok (Cassava) & 79 & 87 & 30 Tschad & 230 & Malawi \\
\hline Zuckerrohr & 524 & 486 & 120 Ceylon & 2200 & Hawaii \\
\hline Zuckerrübe & 200 & 247 & 150 Pakistan & 450 & Israel \\
\hline Kohl & - & - & 40 Uruguay & 670 & Belgien \\
\hline Tomaten & 19 & 198 & 30 Benin & 1420 & Dänemark \\
\hline Zwiebeln & 10 & 131 & 20 Thailand & 530 & Belgien \\
\hline Speisebohnen & 10 & 5 & 2 Portugal & 25 & Belgien \\
\hline Trockenerbsen & 10 & 9 & 6 Lesotho & 32 & Belgien \\
\hline Kichererbsen & 7 & 6 & 3 Portugal & 16 & Ägypten \\
\hline Pferdebohnen & 5 & 11 & 5 Portugal & 39 & Argentinien \\
\hline Linsen & 1 & 6 & 3 Tunesien & 16 & Ägypten \\
\hline Sojabohnen & 37 & 12 & 5 Zimbabwe & 22 & Italien \\
\hline Erdnüsse in der Schale & 15 & 9 & 2 Kongo-Brazz. & 34 & Israel \\
\hline Sonnenblumen & 8 & 10 & 4 Tansania & 17 & Jugoslawien \\
\hline Rapssaat & 5 & 6 & 4 Indien & 26 & Holland \\
\hline Leinsaat & 4 & 5 & 2 Tunesien & 14 & Neuseeland \\
\hline Sesam & 2 & 3 & 1 UdSSR & 10 & Ägypten \\
\hline Baumwolle & 12 & 3 & 1 Togo & 12 & Israel \\
\hline Jute und Kenaf & 3 & 13 & 8 Indien-Kenaf & 11 & VR China-Jute \\
\hline Hanf & 0 & 5 & 4 UdSSR & 23 & Taiwan \\
\hline Flachs & 1 & 4 & 2 Türkei & 13 & Holland \\
\hline
\end{tabular}


Man darf nämlich nicht übersehen, daß das HolzErzeugungspotential der Entwicklungsländer zwar relativ groß ist, größer als das der anderen Nationengruppen. Es wird jedoch auch heute noch nur zu einem Bruchteil ausgenutzt. Daran ist auch eine mangelhafte Entwicklung des Binnenmarktes in den Entwicklungsländern schuld.

$\mathrm{Daß}$ die Rolle der Entwicklungsländer in der 1 a n d wirtschaftlichen Produktion hingegen durchaus kritisch ist, geht aus der Tab. 2 hervor. Zwar ist die biologische Primär-Produktivität vieler Entwicklungsländer, v. a. der in den „ökologisch benachteiligten Tropen" (WEISCHET 1977) gelegenen, sehr hoch. Wie z. B. aus der Karte von LIETH (1975, fig. 2-1) hervorgeht, erreicht sie z. B. im Amazonasgebiet, Teilen Mittelamerikas, Äquatorialafrikas und Insulinde weitgehend über $800 \mathrm{~g}$ Kohlenstoff $/ \mathrm{m}^{2} / \mathrm{a}$; für das landwirtschaftliche Ertragspotential sagen aber solche Zahlen erfahrungsgemäß nicht viel aus.

Denn, wie bereits aus wenigen Beispielen ersichtlich ist, wird das $\mathrm{M} \mathrm{a} \mathrm{x} \mathrm{i} \mathrm{m} \mathrm{u} \mathrm{m} \mathrm{der} \mathrm{landwirtschaftlichen}$ und gärtnerischen $\mathrm{Produktion}$ von den e n t w i ckelt e n Ländern erbracht, und zwar auch unter $z$. T. ungünstigen klimatischen Bedingungen, und außerdem sind die Einzelerträge dort meist sehr hoch (Tab. 2). Naturräume, von denen eine hohe Leistung, z. B. bezüglich der Getreide-, Hackfrucht-, Reisproduktion etc. erwartet werden könnte, hinken statt dessen stark zurück, und ganze Volkswirtschaften aus solchen Regionen sind, $z$. T. auch wegen durch u. E. falsche politische Vorgaben entstandener Leistungsschwäche, auf wenige starke, gut durchorganisierte und von der privatwirtschaftlichen Kreativität, Innovationsfreude und technischen Vollkommenheit lebende Produktionsgebiete, wie z. B. die USA, angewiesen. Immerhin war die Zuwachsrate der landwirtschaftlichen Produktivität mit 2,1\%(1960-1966) in den Entwicklungsländern höher als $(1,8 \%)$ in den Industrie-Ländern (PEARSON et aliae 1969). Daß daraus eine Chance für die Entwicklungsländer zu postulieren ist, liegt auf der Hand. Entscheidend ist jedoch, daß in den Entwicklungsländern

1) zunächst die bodenkundliche (und hydrologischhydrogeologische) Landesaufnahme vorangetrieben werden muß, um die Lokation der einzelnen Bodenstandorte und ihr Potential genau zu ermitteln. Wo entsprechende Institutionen fehlen, müssen sie eingerichtet werden. Quartärforscher sollten in diesen Institutionen nicht fehlen, und diese sollten ihre Tätigkeit nicht auf Abhandlungen über Geomorphologie, Stratigraphie, $\mathrm{Pa}$ läoklimatologie der entsprechenden Länder beschränken sondern sehen, wo die Menschen dieser Region in praktischen Fragen der Schuh drückt.
2) Auf der Basis der geowissenschaftlichen Landesaufnahme muß ein Bodennutzungs-Plan entwikkelt werden, mit Hilfe dessen die richtige Verwendung der Bodenstandorte festgelegt werden kann.

3) Sodann gilt es, die für den entsprechenden Standort richtige Bearbeitungsmethode auszuwählen, den vorteilhaftesten Pflanzentyp, die vernünftigste Fruchtfolge festzulegen.

4) Besonderes Augenmerk ist auf die Ausmerzung von Bearbeitungsmethoden zu legen, die Bodenabtrag und Bodenverarmung hervorrufen (z. B. Branntkulturen, Überweidung). Der Kampf gegen die Bodenerosion muß mit sachgemäßen wasserwirtschaftlichen Arbeiten einhergehen, durch die nicht nur Überschwemmungsgefahren, sondern auch Ansätze für die Tiefenerosion erkannt werden.

5) Die Bodenverarmung, die in vielen Entwicklungsländern nicht nur durch einen Abtrag des Oberbodens sondern auch durch Veränderung der Bodenstruktur (Krümelstruktur $\rightarrow$ Einzelkornstruktur) und anderer wichtiger bodenphysikalischer Parameter gekennzeichnet ist, muß nicht nur durch sachgerechten Einsatz richtiger Düngemittel, sondern auch durch Änderung traditionell verwendeter (d. h. falscher) Bewässerungsverfahren - dazu gehört die unsinnige Überflutung bekämpft werden. Ausbildung und Umerziehung sind hier vordringlich.

6) Gewarnt werden muß vor landwirtschaftlicher Überproduktion, Übergrasung, Überbeanspruchung der Aquifere und Oberflächengewässer wegen egoistischer Vermehrung des Weideviehs - eine wichtige Ursache für das Sahel-Problem! -, vor Überproduktion auch in den Wäldern (d. h. vermehrte Abholzung) und allen ähnlichen Maßnahmen, die zwar kurzfristig Naturraumpotential (für einige Egoisten) frei setzen, das Gesamt-Potential aber um so nachhaltiger (zum Schaden der Gesamtheit) beeinträchtigen.

In einer besseren Nutzung ihrer Böden, die vernünftigen Bodenschutz beinhaltet, sollten viele, v. a. mit mineralischen Bodenschätzen nicht gesegnete Entwicklungsländer ihre größte Chance sehen.

Die rasche Zunahme der Bevölkerung der Erde wäre keine Gefahr, wären die Entwicklungsländer (und einige der Ostländer) heute in der Lage, ihr Bodenpotential - im Einklang mit dem Natur- (d. h. Wasser-, Nährstoff-, biologischen etc.) Haushalt optimal zu nutzen. Bleibt hingegen die Reaktion auf die Bevölkerungsexplosion im Hinblick auf die Bereit- 
stellung von Wasser und Nahrungsmitteln eine Aufgabe für die wenigen eingefahrenen Produktionsgebiete, so ist abzusehen, daß die Grenze des Wachstums tatsächlich bald erreicht ist.

\section{Ingenieurgeologische Probleme}

Fast täglich erinnern uns die Massenmedien daran, daß wir nicht, wie manche Träumer aus der grünen Ecke das offensichtlich glauben, in eine freundliche und idyllische Natur eingebettet sind, die wir deswegen mit Zähnen und Klauen verteidigen sollten. Unser Leben ist stattdessen voller Risiken, und viele davon sind nicht selbstgemacht. Unter diesen natürlichen Hazards, gegen die uns die Kunst der Ingenieure zu schützen versucht, beruhen viele auf Gegebenheiten und Vorgängen, die die Quartärforschung am besten erklären kann. Vernünftige Ingenieurmaßnahmen müssen daher auf geologischen Rat zurückgreifen, und dieser Rat sollte nicht nur aus ingenieurgeologischen sondern vor allem quartärgeologischen Zirkeln kommen.

Man kann diese Empfehlung aus einigen besonders auffälligen Begebenheiten entwickeln:

a) Jeder Küstenbauer an den Gestaden der Nordsee weiß, daß die Berechnung seiner Ingenieurbauten nicht nur auf den normalen bodenmechanischen Größen

- geologisches Profil,

- Mächtigkeit der setzungsempfindlichen Schichten,

- Kompressibilität derselben, basierend auf Korngrößenverteilung, Wassergehalt, Lagerungsdichte usw.,

- Entwässerbarkeit,

- Höhenlage zur Vorflut,

- Scherfestigkeit,

- Quellvermögen der eingebauten Minerale usf.

beruhen darf, daß vielmehr die mit dem postglazialen Meeresspiegelanstieg und $z$. T. mit epirogenen oder auch jungen tektonischen Bewegungen zusammenhängende Küstensenkung berücksichtigt werden muß. Ohne auf diesen Fragenkomplex eingehen zu wollen, kann gesagt werden, daß bei den in den Niederlanden und in Nordwestdeutschland nach verheerenden Sturmfluten vorgenommenen Deicherhöhungen auf diese Bezugsgröße Rücksicht genommen wurde. Quartärforscher waren an ihrer Beschreibung und Größeneinschätzung in hervorragender Weise beteiligt (z. B. DECHEND 1956; FAIRBRIDGE 1961; HAGEMAN 1960; Jelgersma 1966; JelgerSMA \& PANNEKOEK 1960; LINKE 1979; STREIF 1975 u. a.). b) Auch der Deichbau in den von Hochwässern gefährdeten Flußtälern ist ohne Befragung von Quartärforschern schlecht beraten. In vielen Fällen, dort wo die Gliederung und Kartierung der Auelehme mit Verläßlichkeit durchgeführt worden ist, hat der Wasserbau über die meist unzuverlässige kartographische Festlegung der "Gesetzlichen Hochwassergrenze" hinausgehende Informationen über die Marken von Jahrtausendhochwässern erhalten. Daß zu diesen flußgeschichtlichen Arbeiten auch die Geochronologie, Vegetationsgeschichte, Sedimentpetrographie und Bodenkunde Hilfe leisten mußten, daran wird dann bei der Durchführung des betreffenden Ingenieurprojektes meist nicht mehr gedacht.

c) Im Kapitel 1 wurde bereits erwähnt, daß ein großer Teil der Menschheit in Überschwemmungsgebieten wohnt. Jährlich erfahren wir von verheerenden Naturkatastrophen in diesen Räumen. Im Grunde genommen verstößt es gegen jede Vernunft, wenn Menschen $z$. B. in Deltagebieten von Bangladesh überhaupt siedeln. Darüber zu richten, steht jedoch niemand zu: Hier wie an vergleichbaren Orten war die Landnahme eine Verzweiflungstat unterprivilegierter Gruppen. Dennoch sollte der Quartärforscher den oft planlos gegen die Naturkatastrophen agierenden (und damit oft viel Kraft und Geld vergeudenden) Instanzen klar machen, welche quartärgeologischen Vorgänge das Grundmuster dieser Regionen vermitteln, und daß es, wie die Erfahrung der Nordseeküstenbewohner zeigt, sinnlos ist, sich w i d e r die Natur zu stemmen. Man muß deren Regeln kennen und eine Technik suchen, die $\mathrm{m}$ i t der Natur angelegt ist. Reißbrettund Betondamm-Denken bieten keine brauchbaren Lösungen.

d) Neben diesen Beispielen aus dem marinen und fluviatilen Faziesraum soll an die terrestrische Fazies erinnert werden. Unwetterkatastrophen zeigen uns in unregelmäßiger Form die Instabilität mancher Hangbildungen. Mit Talsperren zusammenhängende Bergstürze, Rutschungen, Schlammströme, Hochwasserwellen haben in der letzten Zeit zahlreiche Todesopfer verursacht. An den Bodenabtrag, der in den Alpen als Folge von Tourismus und Sport zu beklagen ist, muß ebenso erinnert werden wie an die Hangbewegungen, die durch den Verkehrswegebau und Wasserbau verursacht werden. Die entsprechenden Dektrationsbildungen in ihren Ursachen, ihrer Bindung u. a. an quartäre Hangsedimente, an die junge Aufwärtsbewegung der entsprechenden Gebirge und die Morphogenese zu erforschen, ist nicht nur Aufgabe der Bodenmechanik sondern der Quartärgeologie mit all' ihren Nachbardisziplinen.

Will man die v. a. für die $\mathrm{Ent}$ wicklungs 1 ä $\mathrm{nder}$ wichtigen Aufgaben der quartärgeologisch untermauerten Ingenieurgeologie besonders 
herausstellen, wie das auch in den vorherigen Kapiteln geschehen ist, so muß man auch an dieser Stelle die für die Anlage von $\mathrm{T}$ a $1 \mathrm{~s}$ p e r r e n notwendigen topographischen, hydrologischen, geologischen und ingenieurwissenschaftlichen Aufgaben erwähnen. Praktisch sind in den meisten Entwicklungsländern - abgesehen vielleicht von einigen Inseln und den absoluten Wüstengebieten - Lokalitäten für Talsperren ausweisbar und dementsprechend auch mehrere Projekte bereits verwirklicht worden oder im Gange. Nicht übersehen werden dürfen dabei neben den geogenen Voraussetzungen die klimatischen Verhältnisse (Verdunstung, Rhythmus der Wasserführung etc.), die Kosten/Nutzen-Bedingungen der hydroelektrischen Kraftwerke, der Irrigationsanlagen sowie die Zwangspunkte, die durch den Energietransport gegeben sind.

Weiteres Geopotential ist für D e p o n i e - Zwecke geeignet. Auch hier spielen die bindigen Lockergesteine neben geologischen Festgesteinskörpern und Strukturen eine wichtige Rolle. Man denke an die Tongesteine, die bei Deponien grundwassergefährdender Stoffe benötigt werden! Aber auch die Beurteilung der Sicherheit von $\mathrm{K}$ a v e $\mathrm{r} n \mathrm{e} n, \mathrm{z}$. B. in Salzkörpern, die Abfallstoffe (z. B. von Kernkraftwerken) oder Massengüter (Rohöl-Kavernen) aufnehmen sollen, bedarf oft quartärgeologischer Mitarbeit, v. a. wenn es darum geht festzustellen, ob ein Salzstock sich noch bewegt, an den hydrologischen Kreislauf angeschlossen ist oder nicht (vgl. das Projekt Gorleben, PTB 1983, ANONYMUS 1983, DUPHORN 1984 u. a.). Das Vorhandensein von Möglichkeiten der Verbringung von kommunalem Müll, Klärschlamm, Abwässern, Gülle, Industrie-Giftmüll in oder auf undurchlässige Horizonte bedarf in vielen Fällen genauester quartärgeologischer Voruntersuchung.

\section{Zur Umwelt- und Planungsgeologie}

Die Umweltgeologie ist der Teil des Fachgebietes, der die geowissenschaftlichen Aspekte der Umweltforschung vertritt, die Planungsgeologie ihre für Landesplanung und Raumordnung relevante Sektion.

In beiden, eng zusammenhängenden Bereichen - sie sind nicht oder nur in bestimmten Teilen ident mit dem nach Auffassung des Autors verschwommen und unklar definierten Teilfach "Anthropogeologie" (KASIG 1983) - liegen eine Fülle von Aufgaben und Möglichkeiten für die Quartärforscher, was an einigen Beispielen demonstriert werden kann.

In der $\mathrm{Umwe}$ m t for s chu ng stehen wir oft vor dem Problem, daß wir durch den Menschen verursachte Veränderungen des Naturraumes nicht oder nur schwer von den natürlichen Veränderungen trennen können. Ein charakteristischer Fall ist die Auelehmbildung, deren durch die Forschungen von NATERMANN (1939, 1942), MENSCHING $(1951,1958)$ u. a. erwiesenen Verursachung durch die mittelalterliche Rodung lange Zeit im Vordergrund gestanden hat, bis die geogene, mit dem postglazialen Meeresspiegelanstieg zusammenhängende Komponente, der wir die älteren holozänen Auelehme verdanken, durch LÜTTIG (1960a, 1960b), STRAUTZ (1962), MÄCKEL (1969), NEUMEISTER (1964) u. a. herausgearbeitet wurde. Hier wurde eine $\mathrm{B}$ e m e s sungsgrund la ge für die Unterscheidung geogen/anthropogen geschaffen, die in vielen anderen Fällen fehlt.

Wichtig ist in dieser Hinsicht auch die $\mathrm{L}$ i $\mathrm{m} \mathrm{n} \mathrm{o-}$ g e o log i e, nicht nur weil Seesedimente oft in phantastischer Weise wie ein erdgeschichtliches Archiv vegetationskundliche, (Beispiel: Ulmenfall) sedimentologische ( $z$. B. Einsetzen der anthropogenen Erosion) und geochemische ( $z$. B. präanthropogene Schwermetallbindung an Mudden) Ereignisse, die für die Beschreibung des „,backgrounds” wichtig sind, festhalten, sondern auch, weil technische Maßnahmen an Seen und die dadurch zu erhoffenden oder zu befürchtenden Veränderungen ohne Kenntnis der natürlichen Dynamik nicht abschätzbar sind (Grahle 1968; Grahle \& H. Müller 1969; LÜTTiG et al. 1980; H. MÜLLER 1969).

Ohne die Vegetationsgeschichte und die $\mathrm{G}$ e o c h r o n o log i e geht es auch hier nicht. Wie sonst wollten wir die Beobachtungen an Eiskernen in Grönland und in der Antarktis (DANSGARD et al. 1971), bei denen geogene Emissionen hohen Ausmaßes zeitlich bestimmt wurden, in ihrer Bedeutung für den Streit um die Verursachung von Vegetationsveränderungen beschreiben können?

Von besonderer Bedeutung sind in diesem Zusammenhang ebenfalls $\mathrm{Ur}$ - und $\mathrm{Fr} u ̈ h \mathrm{ge}$ $\mathrm{s} \mathrm{ch}$ i c h t e wegen der durch sie für die Abwägung der geogenen gegenüber der anthropogenen Umweltveränderungen gesammelten Daten. Überhaupt ist dieses ein Beispiel für die Rolle der Quartärstratigraphie für die Klimaprognostik, auf die beim INQUA-Klimasymposium in Uppsala 1975 (vgl. STARKEL 1976) und durch den Verfasser in seinem Vortrag vor der DEUQUA in Zürich 1982 (Mskrpt. erscheint demnächst) aufmerksam gemacht worden ist, die leider noch zu wenig genutzt wird.

Für die P l a n u n g s g o log i e, deren Beginn mit der Entstehung der $\mathrm{Naturaum}$ pote n ti a lk a r t e n (LÜtTIG 1971, 1972, 1977, 1979; LÜTTIG \& PFEIFFER 1974; BeCKER-PlATEN; LÜTTIG \& MEINE 1979; VON DANIELS \& LÜTTIG 1982) 
gleichgesetzt werden kann, war die Frage des Schutzes oberflächennaher Lagerstätten, also i. w. quartärer Rohstoffe, ein inzwischen auch durch die $\mathrm{L}$ a $\mathrm{g}$ e $\mathrm{r}$ $\mathrm{s}$ t ät t e n s i che r u n g s k a t e (V. STEIN et al. 1981; STEIN \& HofMEISTER 1977; PAULY 1980, 1981; BeCKER-PLATEN \& STEIN 1979) unterstrichener Ansatz, der der angewandten Geologie zu einer neuen Rolle verholfen hat, die v. a. die Quartärgeologen besser mitspielen sollten. Wie man an der verspätet aber auf breiter Front einsetzenden Begeisterung auch anderer Teildisziplinen für diesen Wissenszweig erkennt, ist hier eine Forschungslücke gefüllt worden, die in Zukunft, so ist zu hoffen, auch einer Reihe von geowissenschaftlichen Absolventen im Berufsleben neue Aufgaben verschaffen kann.

Nicht so glücklich ist die Rolle, die die Quartärforscher auf dem Gebiet der R e kult i vi e r u n g s Geologie gespielt haben. Dieses Gebiet, in dem sie wie Bodenkundler, Ingenieurgeologen und Hydrogeologen dringend gebraucht werden und das zurückerobert werden muß, haben sie weitgehend Landschaftsgärtnern, -architekten, Biologen etc. überlassen, deren Rat in vielen Fällen zu absurden Maßnahmen und überhaupt zu einer Ideologisierung der Rekultivierung geführt hat. In diesem Zusammenhang ist auch die sogenannte Renaturierung zu nennen - ein unsinniger Ausdruck! - . Daß bei der Frage der Wiederherstellung eines natürlichen Zustandes zunächst definiert werden muß, welcher Zustand gemeint ist, und auf welchen Irrtümern entsprechende Forderungen zuweilen beruhen, konnte am Beispiel der Hochmoore gezeigt werden, deren wesentliche Entwicklung in der Natur, v. a. in Norddeutschland, erst nach dem Beginn des Ackerbaues erfolgte (vgl. Lüttig 1978, 1984), weshalb „Renaturierung”, also Herstellung eines Präanthropos-Stadiums (welcher Anthropos ist gemeint?) hier bedeuten würde, daß man die Torflagerstätten beseitigen müßte, was aber gar nicht den Intentionen des Naturschutzes entspricht.

\section{Die Reaktion von Forschung und Lehre}

Aus dem Vorhergesagten ergibt sich eine Fülle von Aufgaben, derer sich Forschung und Lehre auf dem Gebiet der Angewandten Quartärgeologie annehmen sollten.

\section{Aber wie sieht die Wirklichkeit aus?}

$\mathrm{Da}$ ist festzustellen, daß bereits der Begriff „Angewandte Quartärgeologie" nicht Allgemeingut der Quartärforscher ist. Man kann aber auch nicht sagen, daß sie auf die Notwendigkeiten nicht reagiert hätten. Es stimmt auch nicht, daß die Mehrzahl der Geowissenschaftler die Probleme des Mannes auf der Straße nicht sieht.
Die bereits erwähnte IFAQ in Brüssel, die Trainingskurse der ECAFE in Kuala Lumpur, bei denen sich v. a. unsere niederländischen Kollegen in rühriger Weise um die Experten aus Entwicklungsländern bemühen, und auch einige der von IUGS und UNESCO unterstützten Forschungsprojekte im IGCP-Programm sind dafür erwähnenswerte Beispiele. Nicht vergessen werden sollten die lange Tradition besitzenden Tagungen der Arbeitsgemeinschaft Nordwestdeutscher Geologen, die ja geschaffen wurde, um Wasserbauer mit Quartärgeologen zusammenzubringen. Manche dieser Zusammenkünfte an der Nord-und Ostseeküste haben den sehr wichtigen Dia$\log$ zwischen Wissenschaft und Praxis vertieft. Auch die Quartärforscher, die in den Sektionen Ingenieurgeologie und Hydrogeologie der DGG tätig sind, müssen in diesem Zusammenhang erwähnt werden. Die DEUQUA ist, so scheint es auf den ersten Blick, kein richtiger Platz für die Angewandte Quartärgeologie. Aber der Schein trügt.

Daß das ganze Problem ein für die Geologie allgemeines ist, sollte nicht übersehen werden. Der Verfasser hat darauf bereits bei der DGG (LUTTIG 1984) hingewiesen. Es hängt mit der Bewertung der Geologie der Lockerablagerungen zusammen. Dazu sei das wichtigste aus der vorgenannten Darstellung wiederholt.

- In der praktischen Geologie sind hierzulande mehr als zwei Drittel der gestellten Gutachten-Fragen auf die Geologie der L o cke r a b la ge r u n $\mathrm{g}$ e $\mathrm{n}$ gerichtet. Und in den Beschäftigungsfeldern der praktischen Geologie außerhalb der Hochschule ist der stark auf Lockerablagerungen bezogene Sektor in deutlicher Ausweitung begriffen - wenigstens, was die Aufgaben, aber auch was die Geologen-Stäbe anbelangt - . Dagegen besteht in mehreren alteingesessenen Berufszweigen, wie z. B. in der Montangeologie und Erzlagerstättenkunde, eine starke Häufung von Experten, aber keine Zunahme der Aufträge. Nach den eigenen Erfahrungen und subjektiver Gewichtung derselben war um 1950-1965 ein wesentlich geringerer Prozentsatz an Geologen in Geologie-, Planungs- und Ingenieurbüros mit hydrogeologischen oder ingenieurgeologischen Fragen beschäftigt als heute. Der Großteil der Absolventen, welche die Hochschulen in die Praxis verließen, ging damals zu Bergbauunternehmen (im weitesten Sinne).

Aufgaben und Fragen geologischer Relevanz kommen heute weitaus öfter aus dem Sachbereich der Lockerablagerungen als dem Bereich, den die FestgesteinsGeologen als echte und einzige Geologie betrachteten und $z$. T. auch heute noch ansehen.

Um nicht mißverstanden zu werden, muß, wie bereits aus dem Vergleich Industrieminerale: Metallerze hervorgeht, herausgestellt werden, daß es dem Autor 
nicht um die Konstruktion eines Gegensatzes zwischen Betätigungsbereichen in der Geologie geht, zumal da es fließende Übergänge zwischen Locker- und Festgesteinen gibt und die scharfe Trennung in Präquartär- und Quartärgeologie oder oberflächennahe Bildungen und Untergrund zu schiefen Begriffsbestimmungen führen würde. „Vielmehr handelt es sich um den Versuch, bewußt zu machen, wo der Geologe im täglichen Umgang mit seinen Berufsfragen die Augen zu öffnen beginnen sollte, nämlich dort, wo sein forschender Geist in die Lithosphäre einzudringen beginnt: In den obersten, meist unverfestigten Zentimetern des geologischen Profils, nicht im unzugänglichen und durch den oberflächennahen Dreck und durch mächtige tiefere Schichten verhüllten Erdkern. Daß es auch dort viele Probleme gibt, die es zu erforschen gilt, ist nicht zu übersehen, sollte aber zunächst nicht zur Debatte stehen. Unsere Geologenfamilie ist zwar klein, aber groß genug, daß sich die Mitglieder derselben die Aufgabenfelder aufteilen; und daß man das eine Terrain als besonders beackerungswürdig herausstellt, soll ja nicht heißen, daß man fordert, die anderen sollten brachliegen" (LÜTTIG 1984).

Der Verfasser hat versucht, die Lage wie folgt bildhaft zu machen: Nach den Vorlesungsverzeichnissen der deutschen Hochschulen betreffen nur rund $8 \%$ der an den das Fach Geologie und Paläontologie vollkommen vermittelnden westdeutschen Hochschulen gehaltenen Vorlesungen, Übungen etc. speziell die Lockerablagerungen. Bei einigem Wohlwollen haben $39 \%$ der Veranstaltungen damit zu tuen, $36 \%$ gelten eindeutig den Festgesteinen und $17 \%$ sind anderen Gebieten gewidmet. Dabei ist eine gewisse Unübersichtlichkeit der Vorlesungsverzeichnisse und eine uneinheitliche Zuordnung z. B. der paläontologischen und lagerstättenkundlichen Veranstaltungen zur eigentlichen Geologie zu berücksichtigen. Das Bild ist trotzdem schief, da das G e w i c h t der gehaltenen, in Vorlesungsverzeichnissen ablesbaren Vorlesungen und Übungen durchaus unterschiedlich ist. Ursache ist auch, daß als Folge unseres bereits in den Oberschulen eingeführten, - wenn man höflich sein will, mindestens mit dem Begriff „merkwürdig" zu belegenden - Ausbildungssystems ein Trend und auch die Möglichkeit besteht, diejenigen Veranstaltungen zu wähle $\mathrm{n}$, in welchen Prüfungen abgehalten werden und in welcher ein Schein erworben werden kann. In dieser Hinsicht ist das Gewicht der Lockerablagerungs-Veranstaltungen mit Sicherheit kleiner als das der Festgesteins-Vorlesungen. Hierzu wäre es nötig, auch den Arbeits-Schwerpunkt der für Prüfungen und Scheinvergabe bestimmenden Dozenten in die Statistik zu übernehmen, was die ganze Abschätzung sicherlich noch komplizierter macht.
Ein anderer Weg ist, die Statistik mit Hilfe der vergebenen Diplom- und Doktorarbeiten zu entwickeln. Auch hier ist keine exakte Abschätzung möglich; die Gründe ähneln den obengenannten. Die in den Nachrichten der DGG von den einzelnen Instituten in den letzten zehn Jahren gemeldeten Arbeiten sind keinesfalls flächendeckend. Deswegen ist die nachfolgende Schätzung sicherlich ebenfalls ungenau aber nicht uninteressant. Die vergebenen Arbeiten betreffen danach zu

\section{$17,5 \%$ die Lockerablagerungen,}

$58,5 \%$ die Festgesteine,

$14 \%$ beide Gebiete, oder sind nicht klar zuordenbar, $10 \%$ andere Themen.

Erkennbar ist jedenfalls, daß doch mehr Lockerablagerungs-Arbeiten vergeben werden, als es der Anzahl der Vorlesungen entspricht, was möglicherweise auch daran liegen kann, daß hierbei (teil)finanzierte praktische Aufträge eine Rolle spielen. Ebenfalls scheint oft die Lage des Universitätsortes zum Verbreitungsgebiet von Lockerablagerungen (aus pragmatischen Gründen) eine entsprechende Aufgabe nahegelegt zu haben. Soweit zu Lehre und Ausbildung!

Die Lage auf dem Sektor F o r s c h u n g ist weniger klar statistisch zu beschreiben, und zwar nicht nur mangels geeigneter Erfassungen, sondern auch, weil die Forschung auf dem Gebiet der angewandten Geologie generell $z$. T. durch Aufträge und entsprechende Sachmittel-Bereitstellung beeinflußt wird, wodurch eine gewisse Unübersichtlichkeit entsteht und ein Vergleich zur sogenannten freien Forschung erschwert wird.

\section{Zur Berufsstrategie}

Bereits aus der Schilderung der Lage auf dem Gebiet von Lehre und Forschung ergeben sich zur Berufsstrategie klare Vorgaben. Die Diskrepanz zwischen praxisgerechter Ausbildung und Berufsanforderung ist ersichtlich. Daraus ist zu schließen, daß das A n g e $\mathrm{bot}$ an Ausbildungsmöglichkeiten in der angewandten Quartärgeologie erhöht werden muß. Damit verbunden ist die Notwendigkeit der Umgruppierung der Ausbildungsstätten in bezug auf die Bildungsund Forschungsschwerpunkte, damit auf Lehrkörper, Einrichtung, Ausrüstung, Studienplan usw. E i n Lehrstuhl für Quartärgeologie und ein paar Stellen für adäquate angewandte Geologie in der Bundesrepublik bedeuten eine eklatante hochschulpolitische Fehldisposition.

Aber auch mit einer Verbesserung dieses Zustandes wäre es nicht getan. In erster Linie muß unsere Berufsgruppe, auch damit der vorgenannte Wunsch erfülllt 
und durchgesetzt werden kann, sich etwas mehr zu Aufgaben für „den Mann auf der Straße” bekennen, öffentlichkeitsnäher arbeiten und sich besser als bisher artikulieren.

Es gilt dabei auch, unsere Umwelt nicht nur von der Wichtigkeit, sondern sogar Unerläßlichkeit unseres Berufes zu überzeugen. Das kann man am besten mit harten Zahlen. Wir sollten uns wirklich nicht zu fein vorkommen, Beispiele zu sammeln, aus denen hervorgeht, was ohne Zuhilfenahme quartärgeologischen Rates an Fehlinvestitionen, Material- und Geldvergeudungen, Unfug, technischen Katastrophen, Bankrotten entstanden ist und was unser Rat für den Einzelnen und die Gemeinschaft bedeuten kann. Auch entsprechende systematische Aufklärungsarbeit ist notwendig.

\section{Einige Worte an die Quartärforscher}

Im Kreise der Quartärforscher, die in der DEUQUA vereinigt sind, darf es erlaubt sein, anschließend einige Beobachtungen vorzubringen, die geeignet sein können, uns alle zu einer Standortsüberprüfung zu veranlassen.

Die Quartärforschung ist nach ihrem Wesen und der Zusammensetzung der daran beteiligten Spezialdisziplinen ein Arbeitsgebiet mit stark interdisziplinärem Charakter, ohne feste Definition, mit sehr übergreifendem Ambiente, aber starker Betonung der „,reinen” Forschung. Das hat seine Vorteile, aber auch eine gewisse Uneinheitlichkeit in der Vorgehensweise, viele methodische und nomenklatorische Unschärfen und Ungenauigkeiten, starke Individualität zur Folge, birgt aber auch die Gefahr der unterschiedlichen Sprache, damit des Aneinandervorbeiredens und eines Mangels an Disziplin.

Die Vertreter der randlichen Spezialgebiete, deren Arbeit für die Gemeinschaft ebenso nützlich ist wie die der Kern-Wissenschaften (v. a. der Geologie, Stratigraphie, Petrographie und Geomorphologie), bedürfen einer ständigen Heranführung an die Grundregeln naturwissenschaftlicher Arbeits- und Ausdrucksweise. Viele Unverständnisse, Fehlleistungen, Abartigkeiten, die wir unter den Arbeitsergebnissen finden, beruhen auf einem Mangel an Klassifikation, Normung und Interpretation. Diese Normung muß aber vom Kern der Forschungsgruppe ausgehen, und hier ist - der Autor spricht für die Geologen - ein Vorwurf an diese Gruppe zu machen, da sie sich für die Quartärforschung in zu geringem Maße engagiert. Die Zeiten der die Quartärforschung bestimmenden klassischen Geognosten ist vorbei. Die junge Generation hat vielfach vielgestaltige, nicht nur geognostische sondern praktisch-geologische Aufgaben.
Hier aber liegt die Chance für eine Neubelebung der DEUQUA. Neben der Gruppe von Quartärforschern, denen die reine und hehre Wissenschaft Verpflichtung bleiben soll, bedürfen wir einer Aktivierung der angewandten Quartärgeologie als eines Teils der dem Bürger direkt dienenden und prospektiven, für unsere Zukunft wirkenden, daseinssichernden Wissenschaft.

\section{Schriftenverzeichnis}

Archer, A. A., LUUtTig, G. W. \& SNezhKo, I. I. (1987): Man's Dependence on the Earth. - (Schweizerbart u. Unesco). - [Im Druck].

Becker-Platen, J. D., LUttig, G. \& Meine, K.-H. (1979): Geoscientific Maps for Planning Natural Resources. Forum, 3: 167-177; New York.

- \& Stein, V. (1979): Methodische Fragen der Rohstoffsicherung in Niedersachsen. - Raumforsch. Raumordn., 1979, 6: 273-279; Hannover.

Blankenburg, P. von \& Cremer, H. D. (1971): Handbuch der Landwirtschaft und Ernährung in den Entwicklungsländern. 2. Pflanzliche und tierische Produktion in den Tropen und Subtropen. - Stuttgart (ULMER).

Christanis, K. (1982): Genese und Fazies der Torf-Lagerstätte von Philippi (Griechisch-Mazedonien) als Beispiel der Entstehung einer Braunkohlen-Lagerstätte vom stark telmatischen Typ. - Diss. Braunschweig: 179 S.; Braunschweig.

DANIELS, C. H. vON \& LUUTTIG, G. (1982): Geowissenschaftliche Karten des Naturraumpotentials als Unterlagen für Raumordnung und Landesplanung. - In: Energierohstoffe im Alpen-Adria-Raum; 151-168; Graz (Amt der Steiermärkischen Landesregierung).

DANSGARD, et al. (1971): Climatic record revealed by the Camp Century ice core. In: [TUREKIAN, K. K.]: The Late Cenozoic glacial ages: 37-56, Hartford 1971.

DECHEND, W. (1956): Der Ablauf der holozänen NordseeTransgression im oldenburgisch-ostfriesischen Raum, insbesondere im Gebiet von Jever i. O. - Geol. Jb., 72: 295-314; Hannover.

FAIRBRIDGE, R. W. (1961): Eustatic changes in sea level. Physics and Chemistry of the World, 4: 99-185; New York.

Florschưtz, F. \& Menendez, A. J. (1962): Beitrag zur Kenntnis der quartären Vegetationsgeschichte Nordspaniens. - Veröff. geobot. Inst. Rübel in Zürich, 37: 67-73; Bern.

Gold, O. \& LÜTTIG, G. (1972): Elbistan - Erfolg einer Untersuchung der Türkei auf Braunkohle mit Mitteln der deutschen Technischen Hilfe (Känozoikum und Braunkohlen der Türkei 7). - Braunkohle, 24, 8: 253-268; Düsseldorf. 
GraHLE, H.-O. (1968): Limnogeologische Probleme in Nordwestdeutschland (Geologische Untersuchungen an niedersächsischen Binnengewässern IV). - Z. deutsch. geol. Ges., 117, 2/3: 727-737; Hannover.

- \& MÜller, H. (1969): Das Zwischenahner Meer. (Geologische Untersuchungen an niedersächsischen Binnengewässern, Nr. V). - Oldenb. Jb., 66: 83-121; Oldenburg i. O.

Hageman, B. P. (1960): De holocene ontwikkeling van de Rijn-Maas-Mond. - Geol. en Mijnbouw, 39: 661-670; 's Gravenhage.

Jelgersma, S. (1966): Sea-level changes during the last 10.000 years. - Royal meteorol. Soc. Proc. Intern. Sympos. World Climate: 54-71; London.

- \& PAnnekoek, A. J. (1960): Post-glacial rise of sealevel in the Netherlands. - Geol. en Mijnb., 39, 6: 201-297; 's Gravenhage.

KollbrunNer, C. F. \& Stauber, H. (1973): Unerschöpfliche saubere Wasser- und Energiequellen in Grönland. - 96 S. (Inst. bauwiss. Forsch.); Zürich.

LIETH, H. (1975): Historical Survey of Primary Productivity Research. - In: [LIETH, H. \& WhITTAKER, R. H.]: Primary Producting of the Biosphere: 7-16, Berlin etc. (Springer).

LINKE, G. (1979): Ergebnisse geologischer Untersuchungen im Küstenbereich südlich Cuxhaven. - Probl. Küstenforsch. südl. Nordseegeb., 13: 39-83; Hildesheim.

LUTTIG, G. (1960b): Neue Ergebnisse quartärgeologischer Forschung im Raume Alfeld-Hameln - Elze. - Geol. Jb., 77: 337-390; Hannover.

- (1960d): Zur Gliederung des Auelehms im Flußgebiet der Weser. - Eiszeitalter \& Gegenwart, 11: 39-50; Öhringen/Württ.

- (1971 g): Die Bodenschätze des Nordsee-Küstenraumes und ihre Bedeutung für Landesplanung und Raumordnung. - N. Arch. f. Nieders., 21, 1: 13-25; Göttingen (1972).

- (1972): Naturräumliches Potential I, II und III. - In: Niedersachsen, Industrieland mit Zukunft: 9-10; Hannover (Nds. Min. Wirtsch. öff. Arb.) 1972.

- (1977a): Die Rolle der geowissenschaftlichen Kartographie in der vorausschauenden Umweltforschung. Kartogr. Nachr., 27, 3: 81-89; Bonn-Bad Godesberg.

- (1977b): Gedanken zur Begriffskette „Torfabbau Wiederherrichtung - Renaturierung". - Telma, 7: 195-201; Hannover.

- (1978): Die Entwicklungsländer mit geringem Geopotential - aus der Sicht des Geowissenschaftlers. 174 S.; Hannover (Nds. Landeszentrale polit. Bildg.).

- (1979): Die oberflächennahen Rohstoffe: Wirtschaftsfaktor - Konfliktursache - Forschungsziel. - Erzmetall, 32, 10: 419-422; Stuttgart.
LÜTTIG, G. (1979b): Geoscientific maps as a basis for landuse planning. - Geol. Fören. Stockh. Förh., 101, 1: 65-69; Stockholm.

- (1984): Die Moornutzung für die Balneologie aus der Sicht von Landesplanung und Umweltschutz. - Heilbad u. Kurort, 84/6: 183-188; Gütersloh.

- (1985): Über den „Dreck, der die Geologie verhüllt” (STILle). - Nachr. dt. geol. Ges., 32: 90-105; Hannover.

- et aliae (1980): Geoscience and Environmental Research in the Federal Republic of Germany (Guide to Excursion A 137 of the 26th International Geological Congress, Paris 1980). - In: LUTTIG, G. W. \& CONTRIBU. TORS: General Geology of the Federal Republic of Germany: 61-95; Stuttgart (Schweizerbart).

- \& DANIELS, C. H. vON (1982): Geowissenschaftliche Karten des Naturraumpotentials als Unterlagen für Raumordnung und Landesplanung. - Energierohstoffe im Alpen-Adria-Raum: 151-168; Graz (Steierm. Landesreg.) (1980).

- \& Marinos, G. (1962): Zur Geologie der neuen griechischen Braunkohlen-Lagerstätte von Megalopolis. Braunkohle, 14, 6: 222-231; Düsseldorf.

- \& Pfeiffer, D. (1974): Die Karte des NaturraumPotentials. Ein neues Ausdrucksmittel geowissenschaftlicher Forschung für Landesplanung und Raumordnung. - N. Archiv Nds. 23, 1:3-13; Göttingen.

MÄCKEL, R. (1969): Untersuchungen zur jungquartären Flußgeschichte der Lahn in der Gießener Talweitung. Gießener geogr. Schr., 19, Eiszeitalter u. Gegenwart, 20: 138-174; Öhringen/Württ.

Melidonis, N. G. (1981): Beitrag zur Kenntnis der Torflagerstätte von Philippi (Ostmazedonien). - Telma, 11: 41-63; Hannover.

Mensching, H. (1951b): Die Entstehung der Auelehmdecken in Nordwestdeutschland. - Proc. 3d intern. Congr. Sedimentol.: 193-210; Groningen-Wageningen.

- (1958a): Soil erosion and formation of haugh-loam in Germany. - Comptes Rendu et Rapports Ass. gén. Toronto, 1: 174-180; Gentbrugge.

MÜLLER, H. (1969): Diskordanzen und Umlagerungserscheinungen in holozänen Sedimenten flacher Seen Nordwestdeutschlands. - Mitt. intern. Verein. Limnol., 17: 211-218; Stuttgart.

NAtermanN, E. (1939 b): Zeitbestimmung einer Flußverlegung auf Grund der Auelehmbildung. - Abh. nat. Ver. Bremen, 31: 326; Bremen.

- (1942): Die Änderungen der Wasserstände und ihr Zusammenhang mit der Auelehmbildung im Wesertal. Die Weser, 21, 2: 15-17; Bremen.

Neumeister, H. (1964): Beiträge zum Auelehmproblem des Pleiße- und Elstergebietes. - Wiss. Veröff. deutsch. Inst. Länderkde. n. F., 21/22: 65-131; Leipzig. 
PAULY, E. (1980): Bewertung und Sicherung mineralischer Rohstoffe aus oberflächennahen Lagerstätten in Hessen, Methoden und Stand. - Erzmetall, 33, 4: 222-225; Weinheim.

- (1981): Wege zur gesicherten Versorgung mit heimischen mineralischen Rohstoffen aus oberflächennahen Lagerstätten. - Erzmetall, 34: 511-518; Weinheim.

PeArson, L. B. et aliae (1969): Bestandsaufnahme und Vorschläge zur Entwicklungspolitik. - Bericht der Kommission für Internationale Entwicklung. - $279 \mathrm{~S}$., $160+45$ S.; Wien etc. (Molden).

PICARD, L. (1963): The Quaternary in the Northern Jordan Valley. - Proc. Israel Acad. Sci. Hum., 1: 4: 1-34; Jerusalem.

Starkel, L. (1976): Presidential Address. - Newsl. Stratigr. 5, 1: 3-5; Berlin, Stuttgart.

StEIN, V. et aliae (1981 b): Lagerstätten der Steine, Erden und Industrieminerale. Untersuchung und Bewertung (vademecum 2). - Schriftenr. GDMB, 38: 1-248; Weinheim etc.

- \& Hofmeister, E. (1977a): Die Darstellung oberflächennaher Rohstoffvorkommen in Rohstoffsicherungskarten. - Geol. Jb., D 27: 121-132; Hannover.
STRAUTZ, W. (1962): Auelehmbildung und -gliederung im Weser- und Leinetal mit vergleichenden Zeitbestimmungen aus dem Flußgebiet der Elbe. - Beitr. Landespflege, 1: 273-314; Stuttgart.

StreIF, H. (1975): Versuch einer Bilanzierung der Sedimentation im Küstenholozän Ostfrieslands. - Geol. Jb., A 28: 3-14; Hannover.

TietzKe, K. et aliae (1980): The Genesis of the Methane in Lake Kivu (Central Africa). - Geol. Rdsch., 69, 2: 452-472; Stuttgart.

[U. N. Department of Economic and SOcial AfFairs] (1976): Ground water in the Western Hemisphere. Nat. Res. Water Ser., 4: 1-337; New York.

WECK, J. (1962): Forstwirtschaftliche Aufgaben in Entwicklungsländern. - 79 S.; Baden-Baden (Lutzeyer).

WeIsCHET, W. (1977): Viel Grün — wenig Nahrung. — Die Umschau, 77, 23: 767-770; Frankfurt a. M.

Manuskript eingegangen am 1. 12. 1985. 